\title{
3D models related to the publication: Morphological and functional changes in the vertebral column with increasing aquatic adaptation in crocodylomorphs
}

\author{
MOLNAR J. L. ${ }^{a^{*}}$, PIERCE S. E. ${ }^{b}$, BHULLAR B.-A. S. ${ }^{c}$, TURNER A. A. ${ }^{\mathrm{d}}$, HUTCHINSON J. R. ${ }^{\mathrm{e}}$
}

${ }^{a}$ Howard University College of Medicine, Department of Anatomy, Washington, DC 20059

${ }^{b}$ Museum of Comparative Zoology and Department of Organismic and Evolutionary Biology, Harvard University, Cambridge, MA 02138

${ }^{c}$ Yale University, Geology \& Geophysics, New Haven, CT 06520

${ }^{d}$ Stonybrook University, Department of Anatomical Sciences, Stony Brook, NY 11794

e Structure \& Motion Lab, Department of Comparative Biomedical Sciences, The Royal Veterinary College, Hawkshead Lane, Hatfield, Hertfordshire, AL9 7TA, United Kingdom

\section{* corresponding author: julia.molnar4@gmail.com}

Abstract: This contribution contains the 3D models described and figured in the following publication: Molnar, JL, Pierce, SE, Bhullar, B-A, Turner, AH, Hutchinson, JR (2015). Morphological and functional changes in the crocodylomorph vertebral column with increasing aquatic adaptation. Royal Society Open Science. doi: 10.1098/rsos.150439

Key words: crocodylomorph, vertebrae, axial skeleton, stiffness, range of motion, aquatic adaptation

Submitted 15.10.2015, Accepted 26.10.2015. doi: 10.18563/m3.1.3.e5

(C) Copyright Molnar J. L. 2015

\section{SPECIMEN LIST}

\begin{tabular}{|c|c|c|c|c|c|c|c|}
\hline $\begin{array}{l}\text { M3 id(s) of 3D } \\
\text { model(s) }\end{array}$ & Species & $\begin{array}{l}\text { Repository } \\
\text { institution }\end{array}$ & $\begin{array}{l}\text { 3D data } \\
\text { acquisition } \\
\text { institution }\end{array}$ & $\begin{array}{c}\text { 3D data } \\
\text { acquisition } \\
\text { method }\end{array}$ & $\begin{array}{c}\text { 3D Data } \\
\text { acquisition } \\
\text { facility model }\end{array}$ & $\begin{array}{l}\text { Voxel size of } \\
\text { original 3D } \\
\text { dataset }\end{array}$ & $\begin{array}{c}\text { 3D data } \\
\text { acquisition } \\
\text { operator }\end{array}$ \\
\hline $\begin{array}{l}\text { M3\#45_NHM-PV } \\
\text { R 7562 }\end{array}$ & $\begin{array}{l}\text { Terrestrisuchus } \\
\text { gracilis }\end{array}$ & $\begin{array}{c}\text { Natural History } \\
\text { Museum, } \\
\text { London, UK }\end{array}$ & $\begin{array}{c}\text { Natural History } \\
\text { Museum, } \\
\text { London, UK }\end{array}$ & $\mathrm{X}$-ray $\mu \mathrm{CT}$ & $\begin{array}{l}\text { Metris X-Tek } \\
\text { HMX ST } 225\end{array}$ & $\begin{array}{c}0.06 \times 0.06 \times 0.06 \\
(\mathrm{~mm})\end{array}$ & $\begin{array}{l}\text { F. Ahmed, } \\
\text { J. Molnar }\end{array}$ \\
\hline $\begin{array}{l}\text { M3\#44_AMNH-VP } \\
3024\end{array}$ & $\begin{array}{l}\text { Protosuchus } \\
\text { richardsoni }\end{array}$ & $\begin{array}{c}\text { American } \\
\text { Museum of } \\
\text { Natural History, } \\
\text { New York, USA }\end{array}$ & $\begin{array}{c}\text { Stonybrook } \\
\text { University, New } \\
\text { York, USA }\end{array}$ & X-ray CT & $\begin{array}{c}\text { LightSpeed } \\
\text { VCT }\end{array}$ & $\begin{array}{c}0.39 \times 0.39 \times 0.39 \\
(\mathrm{~mm})\end{array}$ & A. Turner \\
\hline $\begin{array}{l}\text { M3\#46 NHM-PV } \\
\text { OR } 32598\end{array}$ & $\begin{array}{c}\text { Pelagosaurus } \\
\text { typus }\end{array}$ & $\begin{array}{c}\text { Natural History } \\
\text { Museum, } \\
\text { London, UK }\end{array}$ & $\begin{array}{l}\text { Natural History } \\
\text { Museum, } \\
\text { London, UK }\end{array}$ & X-ray $\mu \mathrm{CT}$ & $\begin{array}{l}\text { Metris X-Tek } \\
\text { HMX ST } 225\end{array}$ & $\begin{array}{c}0.08 \times 0.08 \times 0.08 \\
(\mathrm{~mm})\end{array}$ & $\begin{array}{l}\text { F. Ahmed, } \\
\text { J. Molnar }\end{array}$ \\
\hline $\begin{array}{l}\text { M3\#47 NHM-PV } \\
\text { R 2054 }\end{array}$ & $\begin{array}{c}\text { Metriorhynchus } \\
\text { moreli }\end{array}$ & $\begin{array}{c}\text { Natural History } \\
\text { Museum, } \\
\text { London, UK }\end{array}$ & $\begin{array}{c}\text { Royal Veterinary } \\
\text { College, London, } \\
\text { UK }\end{array}$ & X-ray CT & $\begin{array}{c}\text { GE } \\
\text { Lightspeed }\end{array}$ & $\begin{array}{c}0.36 \times 0.36 \times 0.36 \\
(\mathrm{~mm})\end{array}$ & $\begin{array}{c}\text { JR. } \\
\text { Hutchinson }\end{array}$ \\
\hline $\mathrm{M} 3 \# 48$ FNC0 & $\begin{array}{l}\text { Crocodylus } \\
\text { niloticus }\end{array}$ & $\begin{array}{c}\text { Royal } \\
\text { Veterinary } \\
\text { College, } \\
\text { London, UK }\end{array}$ & $\begin{array}{c}\text { Royal Veterinary } \\
\text { College, London, } \\
\text { UK }\end{array}$ & X-ray CT & $\begin{array}{c}\text { GE } \\
\text { Lightspeed }\end{array}$ & $\begin{array}{c}0.41 \times 0.41 \times 0.41 \\
(\mathrm{~mm})\end{array}$ & $\begin{array}{c}\text { JR. } \\
\text { Hutchinson }\end{array}$ \\
\hline
\end{tabular}




\section{METHODS}

The 3D surfaces were extracted semi-automatically within Materialise Mimics (Materialise Inc. (www.materialise.com/ mimics); Leeuwen, Belgium) using the segmentation threshold selection tool. The 3D surface models are provided in .stl format, and can therefore be opened with a wide range of freeware.

\section{ACKNOWLEDGEMENTS}

Grant sponsor: Natural Environment Research Council

(NERC). Grant numbers: NE/G005877/1 and NE/K004751/1

\section{BIBLIOGRAPHY}

Molnar, J. L., Pierce, S. E., Bhullar, B.-A., Turner, A. H., Hutchinson, J. R., 2015. Morphological and functional changes in the crocodylomorph vertebral column with increasing aquatic adaptation. Royal Society Open Science. doi: 10.1098/rsos.150439. 